\title{
MATERIALS FOR SHIELDING ASTRONAUTS FROM THE HAZARDS OF SPACE RADIATIONS
}

J. W. Wilson*, F. A. Cucinotta**, J. Miller***, J. L. Shinn*, S. A. Thibeault*, R. C. Singleterry*, L. C. Simonsen*, and M. H. Kim****

*NASA Langley Research Center, Hampton, VA 23682, john.w.wilson@larc.nasa.gov

**NASA Johnson Space Center, Houston, TX 77058

***DOE Lawrence Berkeley National Laboratory, Berkeley, CA 94720

****NRC/NAS Fellow NASA Langley Research Center, Hampton, VA 23682

\begin{abstract}
One major obstacle to human space exploration is the possible limitations imposed by the adverse effects of long-term exposure to the space environment. Even before human spaceflight began, the potentially brief exposure of astronauts to the very intense random solar energetic particle (SEP) events was of great concern. A new challenge appears in deep space exploration from exposure to the low-intensity heavy-ion flux of the galactic cosmic rays (GCR) since the missions are of long duration and the accumulated exposures can be high. Because cancer induction rates increase behind low to rather large thickness of aluminum shielding according to available biological data on mammalian exposures to GCR like ions, the shield requirements for a Mars mission are prohibitively expensive in terms of mission launch costs. Preliminary studies indicate that materials with high hydrogen content and low atomic number constituents are most efficient in protecting the astronauts. This occurs for two reasons: the hydrogen is efficient in breaking up the heavy GCR ions into smaller less damaging fragments and the light constituents produce few secondary radiations (especially few biologically damaging neutrons). An overview of the materials related issues and their impact on human space exploration will be given.
\end{abstract}

\section{INTRODUCTION}

The ionizing radiations in space affecting human operations are of three distinct sources and consist of every known particle including energetic ions formed from stripping the electrons from all of the natural elements. The radiations are described by field functions for each particle type over some spatial domain as a function of time. The three sources of radiations are associated with different origins identified as those of galactic origin (galactic cosmic rays, GCR), particles produced by the acceleration of solar plasma by strong electromotive forces in the solar surface and acceleration across the transition shock boundary of propagating coronal mass ejecta (solar energetic particles, SEP), and particles trapped within the confines of the geomagnetic field. The GCR constitutes a low level background which is time invariant outside the solar system but is modulated over the solar cycle according to changes in the interplanetary plasma which excludes the lower energy galactic ions from the region within several AU of the sun [1]. The SEP are associated with some solar flares which produce intense burst of high energy plasma propagating into the solar system along the confines of the sectored interplanetary magnetic field [2] producing a transition region in which the SEP are accelerated. SEP have always been a primary concern for operations outside the Earth's protective magnetic field and could deliver potentially lethal exposures over the course of several hours [3]. The trapped radiations consist mainly of protons and electrons within two bands centered on the geomagnetic equator reaching maximum intensity at an altitude of $3,600 \mathrm{~km}$ followed by a minimum at 7,000 km and a second very broad maximum at $10,000 \mathrm{~km}[4]$. The trapped radiations have limited human operations to altitudes below several hundred kilometers and potentially lethal exposures are obtained over tens of hours in the most intense regions. Low inclination orbits are shielded from extraterrestrial radiations by the geomagnetic field and are mainly exposed to the trapped environment. Inclinations above $45^{\circ}$ are sufficiently near the geomagnetic poles for which GCR and SEP exposures can be significant. Indeed, about half of the expected exposures of the International Space Station (ISS) in its inclined orbit of $51.6^{\circ}$ will be from GCR [5]. 
In the usual context, shielding implies an alteration of the radiations through interactions with intervening materials by which the intensity is decreased. This understanding is to some degree correct in the case of the relatively low energy particles of the SEP and the trapped radiations wherein the energy deposited in astronaut tissues can be easily reduced by adding shield material. As one would expect, some materials are more effective than others as the physics of the interactions differ for various materials. The high energies associated with the GCR are distinct in that the energy absorbed in astronaut tissues is at best unchanged by typical spacecraft shielding configurations and use of some materials in spacecraft construction will even increase the energy absorption by the astronaut. For GCR, one must abandon the concept of "absorbing" the radiation by use of shielding. The protection of the astronaut in this case is not directly related to energy absorption within their body tissues but rather depends on the mechanism by which each particle type transmitted through the shield results in biological injury. Even though the energy absorption by the astronaut can be little affected, the mixture of particle types is strongly affected by the choice of the intervening shield material. Knowledge of the specific biological action of the specific mixture of particles behind a given shield material and the modification of that mixture by choice of shield materials is then a critical issue in protecting the astronaut in future human exploration and has important implications on the design and operation of ISS.

Understanding the biological effects of GCR behind intervening material is then key to protection in future NASA activity in either ISS or deep space. As yet no standards on protection against GCR exposures have been promulgated since insufficient information exists on biological effects of such radiations [6,7]. The most important biological effect from GCR exposure of which we are currently aware is cancer induction which relates to mutation and transformation (a specific mutation) events in astronaut tissues. Our knowledge of radiation carcinogenesis in humans is for gamma ray exposures for which excess career risk is proportional to tissue dose (energy absorbed per unit mass) accumulated at low dose rates. Although insufficient data exists to estimate astronaut cancer risks from the GCR high charge and energy (HZE) ions, there exist relatively detailed data on the biological response of several systems including survival, neoplastic transformation, and mutation in mammalian cells and Harderian gland tumor induction in mice. Other biological effects may come to light as exposure of living systems to high energy heavy ion beams continues to be studied. We will discuss the available response models in light of the design criteria used for ISS and the implications for materials research. For further discussion of these issues see "Shielding Strategies for Human Space Exploration" [8]. In the present paper, we review the GCR environment and discuss the issues of shield design in the context of developing a strategy for reducing the health risks of astronauts in future missions. In particular we will examine the role of materials research and development in controlling astronaut health risks from exposure to ionizing radiation in space.

\section{GCR AND BIOLOGICAL RESPONSES}

The galactic cosmic rays consist mainly of nuclei (ions) of the elements of hydrogen thru nickel. The energy spectra are broad and extend from tens to millions of MeV (figure 1). The most important energies for protection lies near maximum intensities from a few hundred to several thousand $\mathrm{MeV} /$ nucleon (a nucleon is the name given to neutrons and protons of which the ions are composed). The salient feature of these radiations is that a significant number of these particles have high charge which affects the means by which energy is transferred to tissues. Their ion tracks seen in nuclear emulsion are shown in figure 2. The optical density (related to energy deposited) of the track increases as the ion charge squared and the intensity and the lateral extent of the track depend on the ion velocity. The tracks in the figure are for approximately 400

$\mathrm{MeV} /$ nucleon ions. Considering that the mammalian cell nucleus size is 

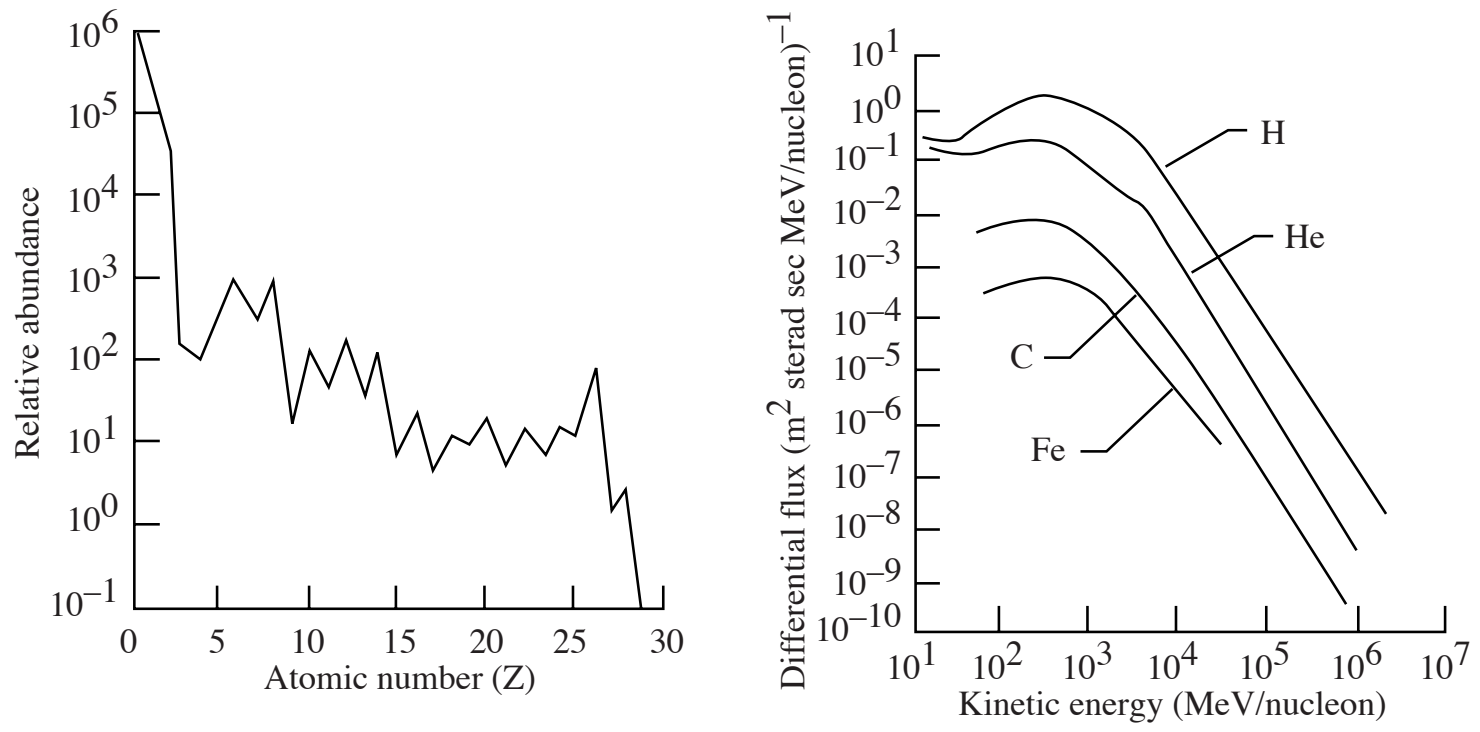

Figure 1. Relative abundances from Mewaldt [9] and selected energy spectra from Simpson [10] for galactic cosmic ray nuclei.

Figure 2. Cosmic-ray ion tracks in nuclear emulsion. (McDonald 1964). 
several micrometers, it is clear that the passage of a single iron ion through the cell nucleus is a potentially devastating event. The protection standards applied to ISS are those recommended for Space Station Freedom scheduled for a low inclination orbit in which GCR exposures were minimal [6]. These standards were adapted, in part, from those used in the nuclear industry for mainly low energy radiations where ion tracks have very limited lateral extents and biological responses are characterized by mainly the energy lost per ion path length (linear energy transfer, LET). The enhanced effectiveness of high LET radiation to cause cancer for a given absorbed energy is given by an LET dependent quality factor [11] as shown in figure 3. The excess cancer risk is then assumed proportional to the dose equivalent which is the product of quality factor and dose. Although little data exists on human exposures from HZE radiations, the limited studies in mice and mammalian cell cultures allow evaluation of the effects of track structure on shield attenuation properties and evaluation of the implications for dosimetry. The most complete HZE exposure data sets for mammalian cells have been modeled including the mouse embryo cells C3H10T1/2 for the survival and neoplastic transformation data of Yang et al.[12, 13], the hybrid hamster cells V79 for the survival and mutation data of various groups [14], and the mouse Harderian gland tumor data of Alpen et al. $[15,16]$. Model results for the Harderian gland tumor data are shown in figure 4 in comparison with data from Alpen et al. [16]. The Harderian target cell initiation cross section (the initiating event in tumor formation is thought to be neoplastic transformation) is shown in figure 5 and compares closely with the transformation cross section found for the C3H10T1/2 cell transformation data of Yang et al. [13]. The most notable feature of the cross sections in figure 5 are the multiple values for a given LET which implies the corresponding relative biological effectiveness (RBE) is dependent not only on the LET but also the ion type. This fact is at variance with the latest ICRP recommended quality factor [11] which is a defined function of only the LET (figure 3 ).

Track structure related events are difficult to study in whole animals since the local environment within an animal varies across the organ under study and is modified by the surrounding tissues. Cell cultures can be used to better control the local environment and provide an improved system for track structure studies. Among the best studied cell systems is the hybrid hamster cell V79 for survival and mutation end points. The model of the V79 system is shown in comparison with data from various groups in figure 6 . As we shall see, these track

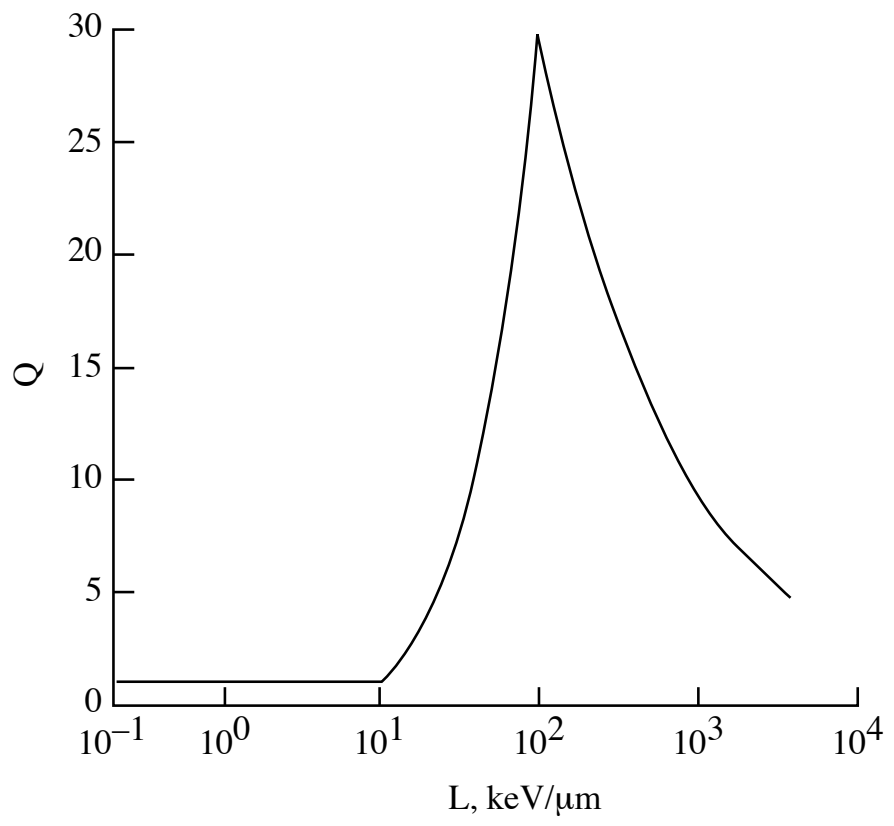

Figure 3. ICRP-60 Recommended Quality Factor. 

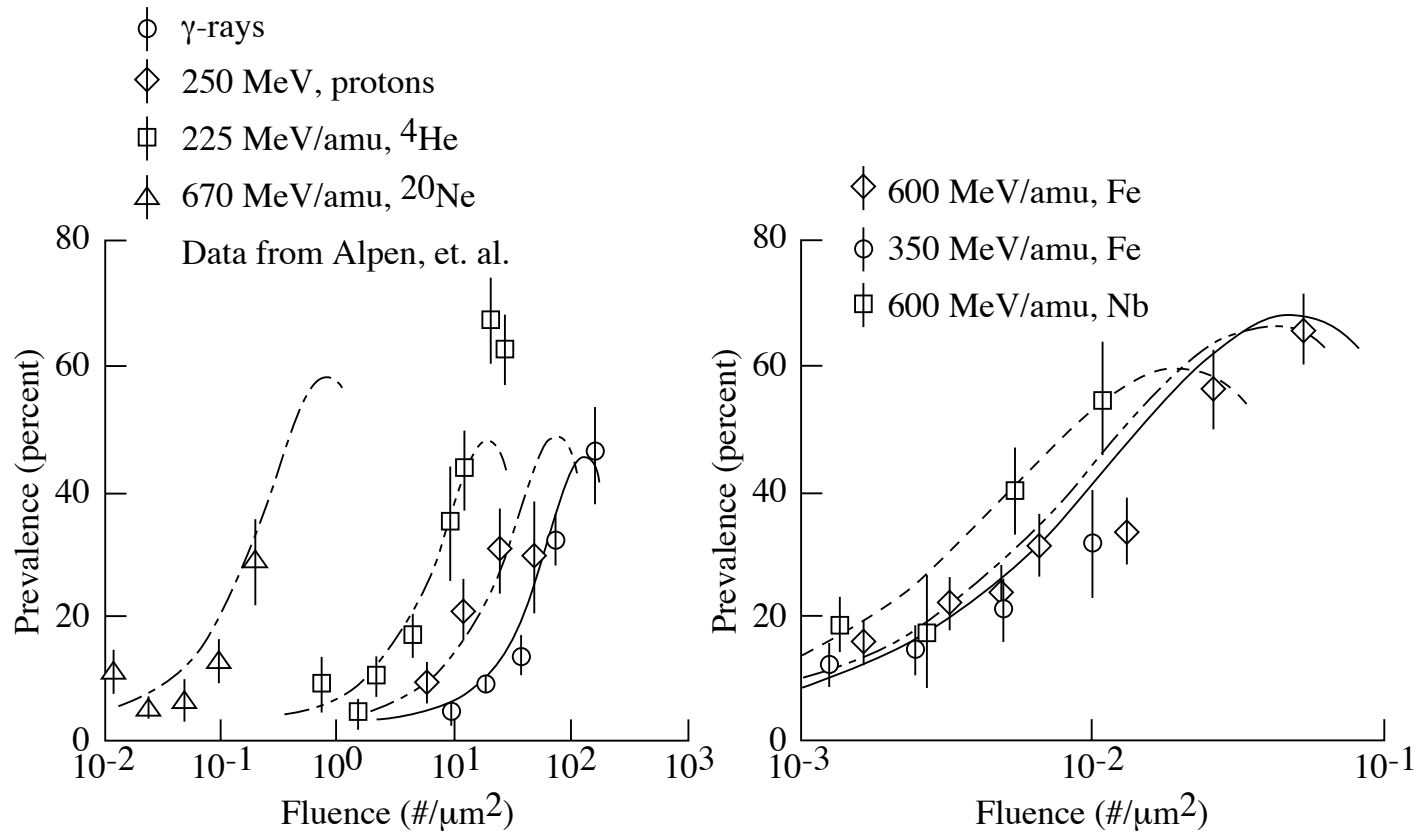

Figure 4. Fluence response for Harderian gland tumors for several radiation types.

structure related features have important implications for attenuation of biological effects within spacecraft shielding materials.

\section{SHIELDING METHODOLOGY}

The specification of the interior environment within a spacecraft and evaluation of the effects on the astronaut are at the heart of the radiation protection problem. The Langley Research

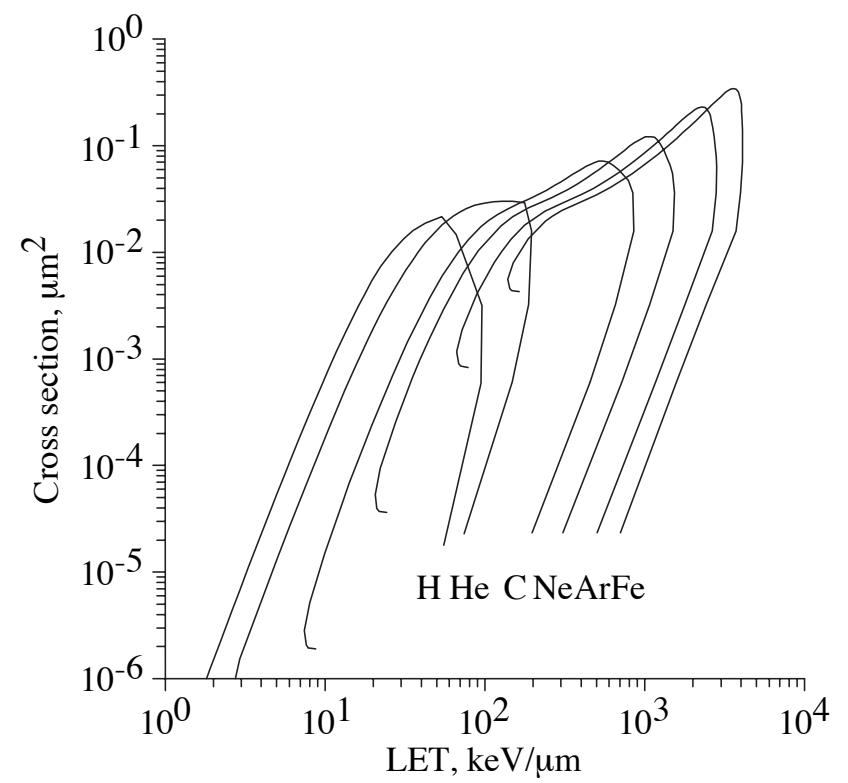

Figure 5. Harderian gland cell initiation cross section obtained from fits to the Alpen et al. data. 

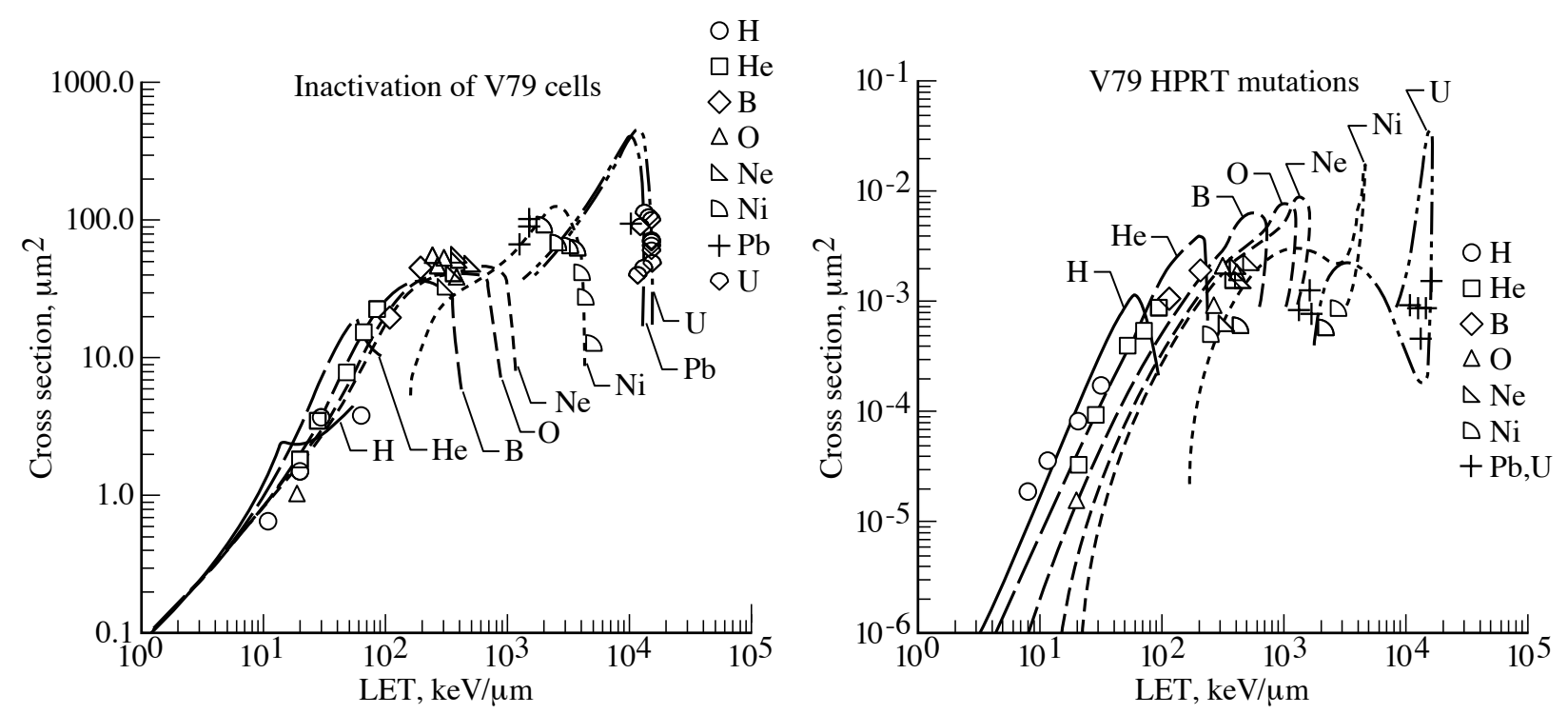

Figure 6. Track structure effects in the V79 cross sections for (a) inactivation and (b) HPRT mutation.

Center has been developing such techniques. The relevant transport equations are the linear Boltzmann equations for the flux density $\phi_{\mathrm{j}}(\mathbf{x}, \Omega, \mathrm{E})$ of type $\mathrm{j}$ particles as

$$
\Omega \cdot \nabla \phi_{\mathrm{j}}(\mathbf{x}, \Omega, \mathrm{E})=\sum \int \sigma_{\mathrm{jk}}\left(\Omega, \Omega^{\prime}, \mathrm{E}, \mathrm{E}^{\prime}\right) \phi_{\mathrm{k}}\left(\mathbf{x}, \Omega^{\prime}, \mathrm{E}^{\prime}\right) \mathrm{d} \Omega^{\prime} \mathrm{d} \mathrm{E}^{\prime}-\sigma_{\mathrm{j}}(\mathrm{E}) \phi_{\mathrm{j}}(\mathbf{x}, \Omega, \mathrm{E})
$$

where $\sigma_{\mathrm{j}}(\mathrm{E})$ and $\sigma_{\mathrm{jk}}\left(\Omega, \Omega^{\prime}, \mathrm{E}, \mathrm{E}^{\prime}\right)$ are the media macroscopic cross sections. The $\sigma_{\mathrm{jk}}\left(\Omega, \Omega^{\prime}, \mathrm{E}, \mathrm{E}^{\prime}\right)$ represent all those processes by which type k particles moving in direction $\Omega^{\prime}$ with energy $\mathrm{E}^{\prime}$ produce a type j particle in direction $\Omega$ with energy E. Note that there may be several reactions which produce a particular product, and the appropriate cross sections for equation (1) are the inclusive ones. The total cross section $\sigma_{j}(\mathrm{E})$ with the medium for each particle type of energy $\mathrm{E}$ may be expanded as

$$
\sigma_{j}(E)=\sigma_{j, a t}(E)+\sigma_{j, e l}(E)+\sigma_{j, r}(E)
$$

where the first term refers to collision with atomic electrons, the second term is for elastic nuclear scattering, and the third term describes nuclear reactions. Any realistic calculation must include not only the description of the primary ion and fragment fields but also the resultant secondary radiations formed in collision of the nuclei of the shield and target material as well as the secondary electrons produced in atomic collisions. The microscopic cross sections and average energy transfer are ordered as follows:

$$
\begin{aligned}
& \sigma_{\mathrm{j}, \mathrm{at}}(\mathrm{E}) \sim 10^{-16} \mathrm{~cm}^{2} \text { for which } \Delta \mathrm{E}_{\mathrm{at}} \sim 10^{2} \mathrm{eV} \\
& \sigma_{\mathrm{j}, \mathrm{el}}(\mathrm{E}) \sim 10^{-19} \mathrm{~cm}^{2} \text { for which } \Delta \mathrm{E}_{\mathrm{el}} \sim 10^{6} \mathrm{eV} \\
& \sigma_{\mathrm{j}, \mathrm{r}}(\mathrm{E}) \sim 10^{-24} \mathrm{~cm}^{2} \text { for which } \Delta \mathrm{E}_{\mathrm{r}} \sim 10^{8} \mathrm{eV}
\end{aligned}
$$

This ordering allows flexibility in expanding solutions to the Boltzmann equation as a sequence of physical perturbative approximations. It is clear that many atomic collisions $\left(\sim 10^{6}\right)$ occur in a centimeter of ordinary matter, whereas $\sim 10^{3}$ nuclear coulomb elastic collisions occur per centimeter. In contrast, nuclear reactions are separated by a fraction to many centimeters 
depending on energy and particle type. Special problems arise in the perturbation approach for neutrons for which $\sigma_{\mathrm{j}, \text { at }}(\mathrm{E}) \sim 0$, and the nuclear elastic process appears as the first-order perturbation.

As noted in the development of equation (1), the cross sections appearing in the Boltzmann equation are the inclusive ones so that the time-independent fields contain no spatial (or time) correlations. However, space- and time-correlated events are functions of the fields themselves and may be evaluated once the fields are known. Such correlations are important to the biological injury of living tissues. For example, the correlated release of target fragments in biological systems due to ion or neutron collisions have high probabilities of cell injury with low probability of repair resulting in potentially large relative biological effectiveness (RBE) and associated quality factor. Similarly, the passage of a single ion releases an abundance of low energy electrons from the media resulting in intense fields of correlated electrons near the ion path.

The solution of equation (1) involves hundreds of multi-dimensional integro-differential equations which are coupled together by thousands of energy dependent cross terms and must be solved self-consistently subject to boundary conditions ultimately related to the external space environment and the geometry of the astronaut's body and/or a complex vehicle. In order to implement a solution one must have available the atomic and nuclear cross section data. The development of an atomic/nuclear database is a major task in code development.

\section{$\underline{\text { Transport Coefficients }}$}

The transport coefficients relate to the atomic/molecular and nuclear processes by which the particle fields are modified by the presence of a material medium. As such, basic atomic and nuclear theories provide the input to the transport code data base $[17,18]$ and requires laboratory validation [19]. It is through the nuclear processes that the particle fields of different radiation types are transformed from one type to another. The atomic/molecular interactions are the principal means by which the physical insult is delivered to biological systems in producing the chemical precursors to biological change within the cells. The temporal and spatial distributions of such precursors within the cell system governs the rates of diffusive and reactive processes leading to the ultimate biological effects. The transport coefficients and their evaluation are described elsewhere $[17,18,19]$.

\section{Transport Solution Methods}

The solution to equation (1) can be written in operational form as $\phi=\mathrm{G} \phi_{\mathrm{B}}$ where $\phi_{\mathrm{B}}$ is the inbound flux at the boundary, and $\mathrm{G}$ is the Green's function which reduces to a unit operator on the boundary. A guiding principle in radiation-protection practice is that if errors are committed in risk estimates, they should be overestimates (conservative). The presence of scattering terms in equation (1) provides lateral diffusion along a given ray. Such diffusive processes result in leakage near boundaries. If $\phi_{\Gamma}$ is the solution of the Boltzmann equation for a source of particles on the boundary surface $\Gamma$, then the solution for the same source on $\Gamma$ within a region enclosed by $\Gamma_{\mathrm{o}}$ denoted by $\phi_{\Gamma \mathrm{o}}(\Gamma)$ has the property

$$
\phi_{\Gamma \mathrm{o}}(\Gamma)=\phi_{\Gamma^{+}} \varepsilon_{\Gamma}
$$

where $\varepsilon_{\Gamma}$ is positive provided $\Gamma_{\mathrm{o}}$ completely encloses $\Gamma$. The most strongly scattered component is the neutron field for which an 0.2 percent error results for semi-infinite media in most practical problems [20,21]. Standard practice in space radiation protection replaces $\Gamma$ as required at some point on the boundary and along a given ray by the corresponding $\Gamma_{\mathrm{N}}$ evaluated for normal incidence on a semi-infinite slab. The errors in this approximation are second order in the ratio of beam divergence and radius of curvature of the object [20], rarely exceeds a few percent for space radiations, and are always conservative. The replacement of $\Gamma$ by $\Gamma_{\mathrm{N}}$ as a highly accurate 
approximation [21] for space shielding applications has the added advantages that $\Gamma_{\mathrm{N}}$ is the natural quantity for comparison with laboratory simulations and has the following properties: If $\Gamma_{\mathrm{N}}$ is known at a plane a distance $x_{\mathrm{O}}$ from the boundary (assumed at the origin), then the value of $\Gamma_{\mathrm{N}}$ at any plane $\mathrm{x} \geq \mathrm{x}_{\mathrm{O}}$ is

$$
\mathrm{G}_{\mathrm{N}}(\mathrm{x})=\mathrm{G}_{\mathrm{N}}\left(\mathrm{x}-\mathrm{x}_{\mathrm{O}}\right) \mathrm{G}_{\mathrm{N}}\left(\mathrm{x}_{\mathrm{O}}\right)
$$

Setting $\mathrm{x}=\mathrm{x}_{\mathrm{O}}+\mathrm{h}$, where $\mathrm{h}$ is small and of fixed-step size gives rise to the marching procedures of the HZETRN code [22] used in the present analysis.

\section{IMPACT ON SHIELDING}

As noted in previous sections, the GCR are of high energy and the energy absorbed in tissues behind typical shields used in space are nearly independent of shield thickness. This is not to imply that the environment has not been changed by the material, indeed the number of particles is normally increased and the mixture of types changed dramatically. Thus, the composition of the transmitted radiations can be greatly altered by choice of materials and amount but with little affect on energy absorption rates. Evaluation of the effects of shielding requires solution of the transport equation (1) describing the alterations to the GCR resulting from individual atomic and nuclear reactions. We have used the biological response models and the LET dependent quality factor to investigate the attenuation of biologically damaging radiation within shield materials in the space environment. In terms of dose equivalent for a shield of thickness $x, H(x)$, we find that aluminum structures attenuate radiation effects over most of the range of depths used in human rated vehicles $\left(2-10 \mathrm{~g} / \mathrm{cm}^{2}\right)$ as shown in figure 7 . Thus, dose equivalent reduction may be a misleading indicator of astronaut satfety [23]. In contrast, track structure models show markedly different attenuation characteristics and, in fact, show that a transformed cell is more likely to result by increasing the aluminum shielding in spite of the decreasing dose equivalent as seen in figure 7.

As a further example of the issues we face, the dose equivalent behind three shield materials is shown in Table I. The first shield is aluminum which is typical of many constructions including ISS. The $1.5148 \mathrm{~g} / \mathrm{cm}^{2}$ thickness is that of the JSC TransHab wall design for a combination of polymers and fillers. The value in parenthesis is the performance advantage of
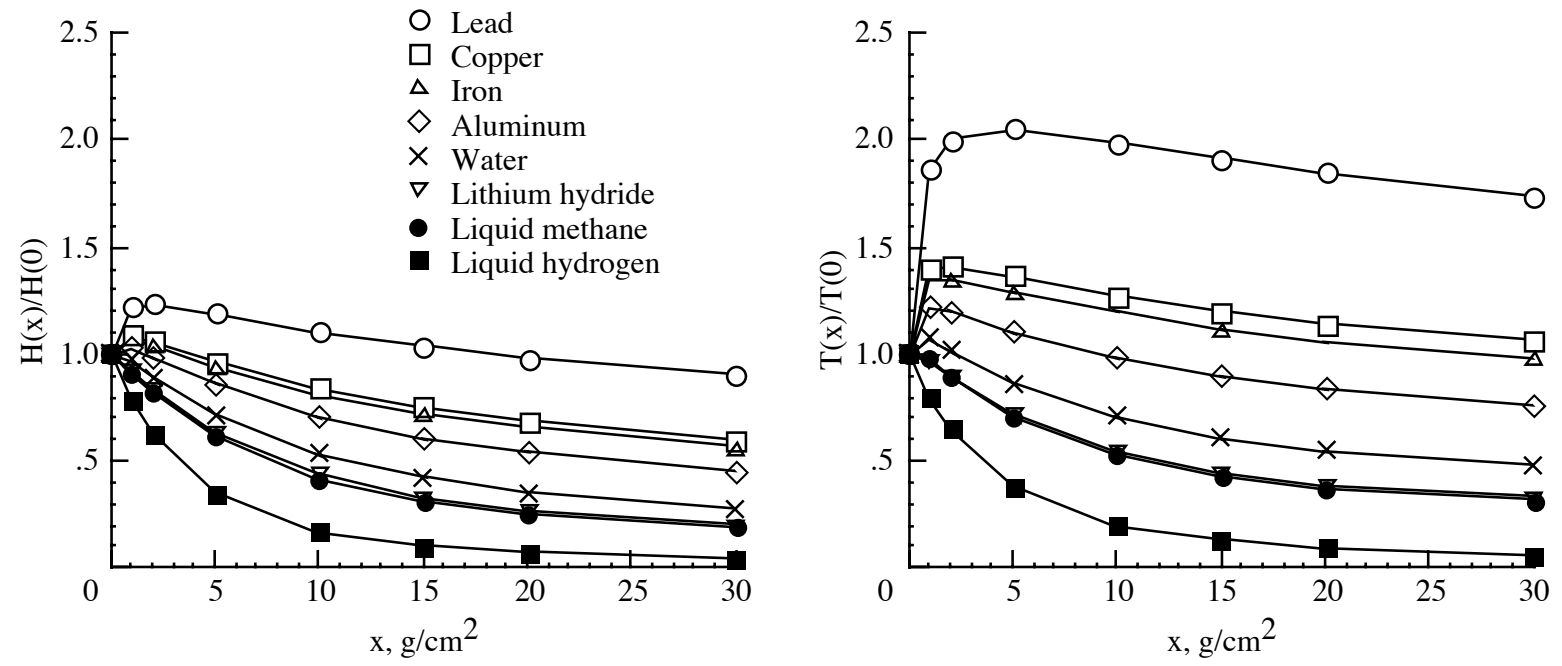

Figure 7. Attenuation of dose equivalent and cell transformation for a one-year GCR exposure at solar minimum behind various shield materials. 
Table I. Annual GCR exposure behind various shields at 1997 solar minimum. Numbers in parenthesis are shield performances relative to aluminum values at the same thickness.

\begin{tabular}{|c|c|c|}
\hline Shield & Dose Equivalent, $\mathrm{cSv}^{*}$ & $\begin{array}{c}\text { Excess Harderian Gland Tumor } \\
\text { Risk, percent* }\end{array}$ \\
\hline $1.5148 \mathrm{~g} / \mathrm{cm}^{2}$ & $130.9(1.00)$ & $3.57(1.00)$ \\
\hline Aluminum & $121.6(1.08)$ & $3.07(1.16)$ \\
\hline TransHab & $113.1(1.16)$ & $2.64(1.35)$ \\
\hline Polyethylene & \multicolumn{2}{|c|}{} \\
\hline $5 \mathrm{~g} / \mathrm{cm}^{2}$ & $113.9(1.00)$ & $3.37(1.00)$ \\
\hline Aluminum & $99.4(1.15)$ & $2.74(1.24)$ \\
\hline TransHab & $86.4(1.32)$ & $2.20(1.54)$ \\
\hline Polyethylene &
\end{tabular}

*Unshielded, dose equivalent is $120 \mathrm{cSv}$ and excess tumor risk is 2.23 percent

the given material compared to aluminum. For example, polyethylene is 16 percent more effective than aluminum in controlling dose equivalent with only $1.5148 \mathrm{~g} / \mathrm{cm}^{2}$ of material. Much larger gains are achieved at $5 \mathrm{~g} / \mathrm{cm}^{2}$ thickness. The $5 \mathrm{~g} / \mathrm{cm}^{2}$ thickness is typical for an area within a human-occupied vehicle loaded with equipment. Modest reductions in dose equivalentare found for all three materials at the $5 \mathrm{~g} / \mathrm{cm}^{2}$ thickness. A mouse carried on the same mission of one year duration will have an excess risk of Harderian gland tumors shown in the last column in Table I. A substantial increase in Harderian tumor risk for shield thickness $x, H G(x)$, is found for both thicknesses of aluminum and great amounts of aluminum are required to reduce the risk. Polyethylene shows substantial improvements in performance for controlling Harderian gland tumor risk at both thicknesses. These findings have important implications for deep space exploration but also for ISS which receives half of its exposure from GCR ions.

\section{MATERIALS RELATED ISSUES}

The biologically based models show complex dependence on radiation quality which is expressed in terms related to the details of the particle track as distinct from the simple LET dependence of the quality factor used in conventional radiation protection practice including ISS. Even the cancer risk attenuation characteristics of spacecraft shield materials are found to be different for the track structure dependent and LET dependent models leading one to conclude that any useful dosimetric technique must take into account these differences as well. Most important in this respect is that LET dependent quality factors overestimate the effectiveness of most shielding materials (see figure 7) and would falsely indicate reduced cancer risk in many applications. Note also that the relative importance of material choices is clearer in the track structure models. This in part results from the fact that aluminum is inherently poor for protecting against tumors in mice induced by the GCR environment. Clearly the relative importance of design alternatives depends critically on our understanding of the biological action of specific radiation components and our ability to evaluate the transmitted radiations through specific shield materials $[7,23]$.

In this regard, the attenuation properties depend on the atomic/nuclear database which has undergone change in recent years. For example, the dose equivalent relative to the unshielded value $\mathrm{H}(\mathrm{x}) / \mathrm{H}(0)$ is shown in figure 8 where $\mathrm{H}(\mathrm{x})$ is the dose equivalent within an aluminum shell of thickness $x$. We normalize the dose equivalent to the unshielded value $H(0)$ to minimize the effects of changing environmental models in the succession of results shown in the figure. The remaining differences are mainly in nuclear models and transport procedures. The curve labeled Letaw et al. [24] is for the database developed by the Naval Research Laboratory in common use 


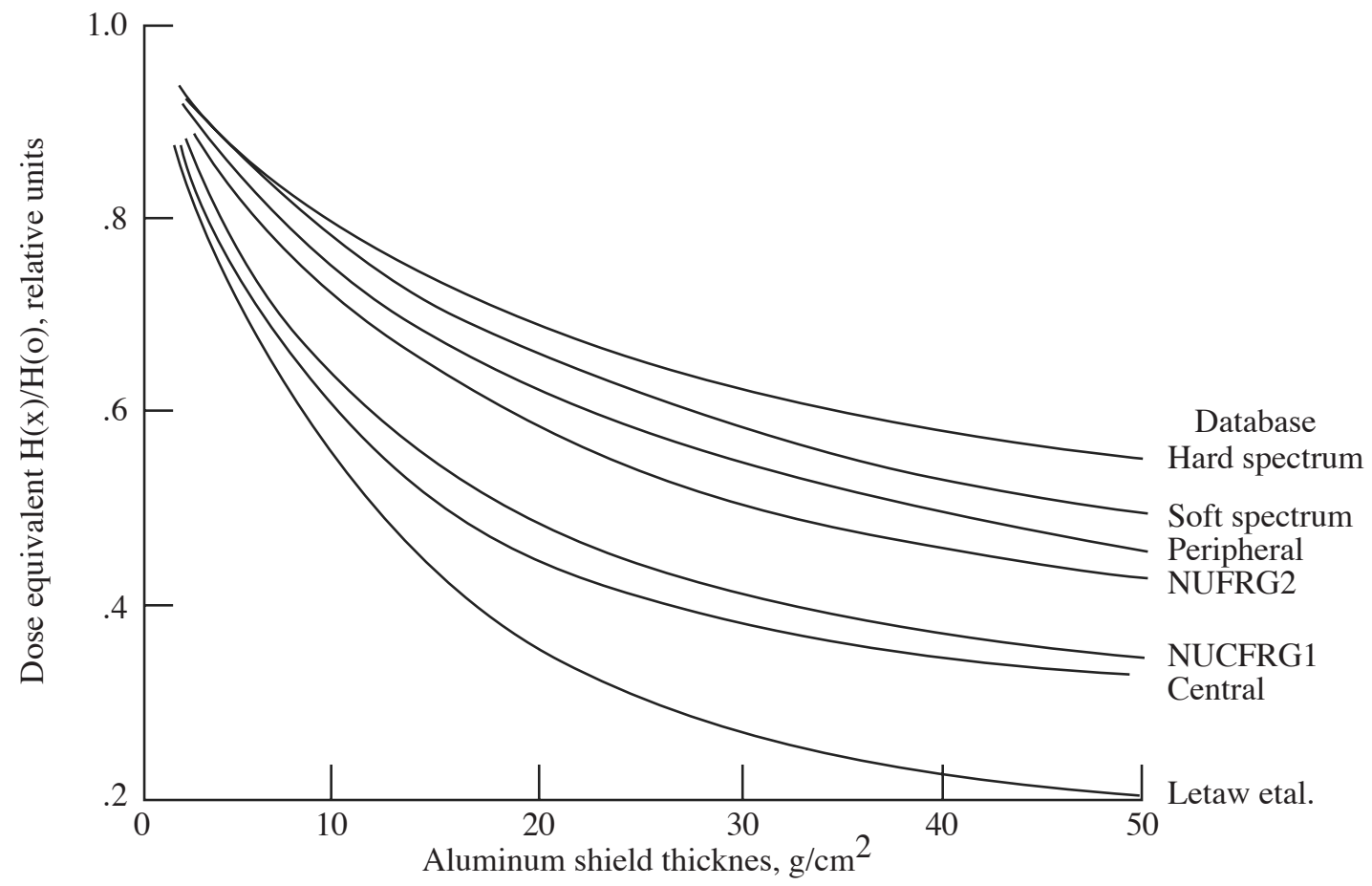

Figure 8. Shield attenuation for solar minimum galactic cosmic ray dose equilvalent resulting from nuclear fragmentation models.

until a few years ago and still used extensively in electronic hardening. NUCFRG1 model is the first database developed by Langley Research Center [25] and already gave higher exposure estimates than the Letaw et al. model for the same environmental model. The NUCFRG2 database is the result of revisions from a set of experiments with iron beams at the Lawrence Berkeley Laboratory Bevalac facility [26]. The hard and soft spectrum results are from direct knockout processes where there is some uncertainty in the knockout spectrum at high energies [27] and are in close agreement with shuttle experiments. Additional revisions in dose equivalent result from environmental model improvements [1]. Although large changes in evaluation of the transmitted spectrum have occurred, it remains an open question how the attenuation will change as new physical processes are added to the transport codes, the nuclear models are improved, and new knowledge of the biological risk comes available [7]. Laboratory validation of the developing database will play an important role in database development [19].

\section{ROLE OF MATERIALS RESEARCH}

In table I we included a performance index as the ratio of dose equivalent in aluminum (Al) to dose equivalent in another material $(\mathrm{M})$ as

$$
\mathrm{P}_{\mathrm{H}}(\mathrm{x})=\mathrm{H}_{\mathrm{Al}}(\mathrm{x}) / \mathrm{H}_{\mathrm{M}}(\mathrm{x})
$$

and a similar performance metric for Harderian gland tumor induction, $\mathrm{HG}_{\mathrm{M}}(\mathrm{x})$. As noted in the table, the predicted performance of a given material relative to aluminum depends on the biological model used and the specific material. For each shield thickness, we can look at the range of variation over the materials in figure 7 and plot the maximal performance values for the two models related to cancer induction (dose equivalent $\mathrm{H}(\mathrm{x})$ and cell transformation, $\mathrm{T}(\mathrm{x})$ ). The maximal performance for each biological model is liquid hydrogen $\left(\mathrm{LH}_{2}\right)$ and the result is shown in figure 9. We have added to figure 9 the relative performance factor based on the Harderian gland tumor risk, $\operatorname{HG}(\mathrm{x})$. Clearly, large performance factors are possible through 


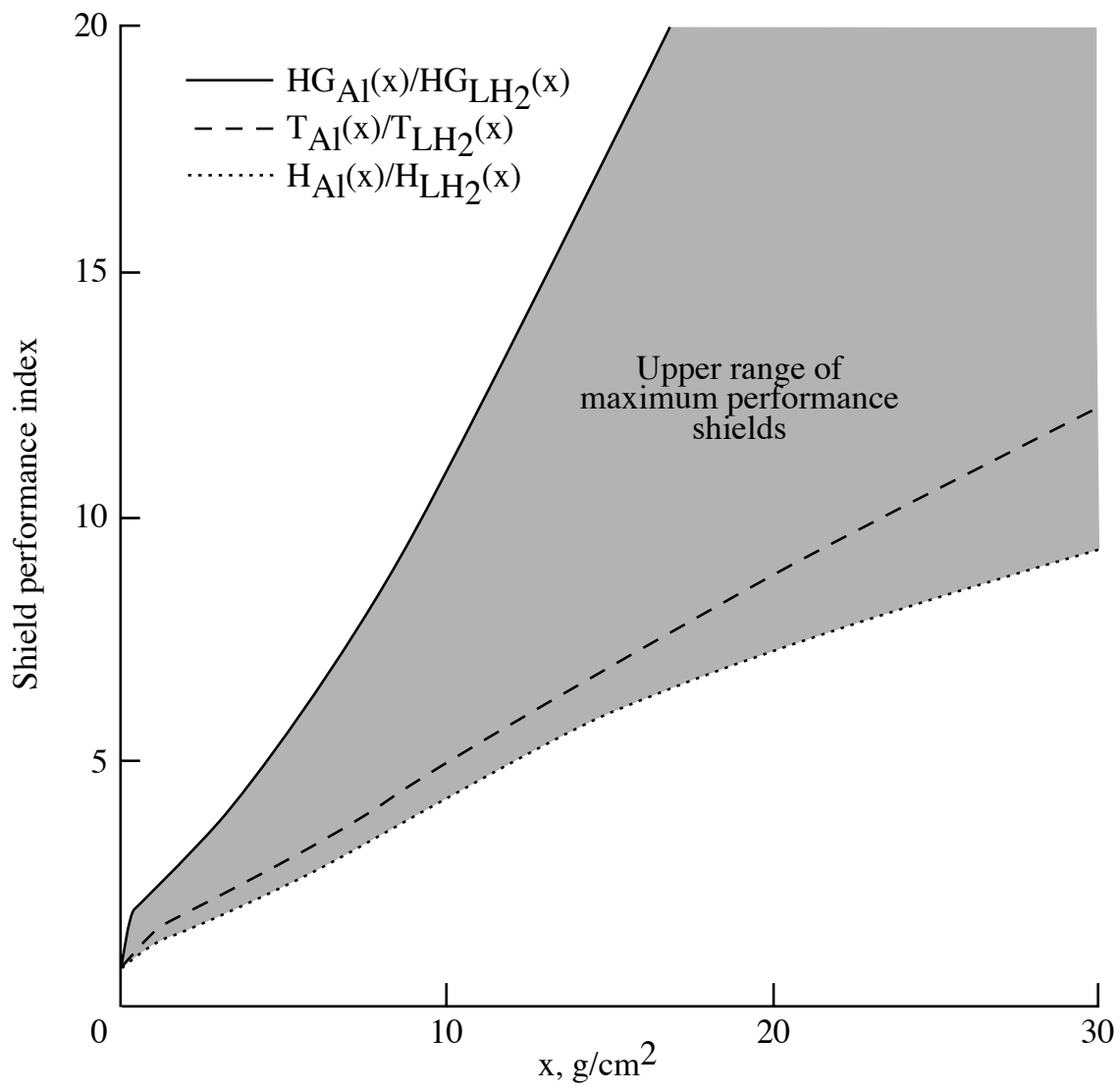

Figure 9. Maximal shield performance factors relative to aluminum with various biological models.

materials development and one would hope to approach the range of maximal performance in figure 9 as closely as possible. Although the actual performance achievable must await an improved understanding of biological response to GCR ions [7], it is clear that high performance shield materials will greatly reduce the mass requirements to protect astronauts in future missions and thereby greatly reduce the associated launch costs.

\section{CONCLUDING REMARKS}

The estimation of shield attenuation characteristics of various materials depends on the details of the biological response model. In that the experimental biological evidence displays clear dependence on other parameters in addition to LET, the adequate shield design for future deep space missions needs to reflect this dependence on these other factors (such as track width). Although accumulated data on biological response to heavy ion exposures allows studies of the relative advantage of material choices, the final design of future mission shielding must await a clearer understanding of the human response to GCR radiations. In the meantime, validation of the transport procedures and identification of high performance shield materials should be the focus of current materials research. In that materials with high hydrogen content yield good shield performance, polymeric materials are expected to play an important role in protecting the astronaut on future missions. As part of the consideration of new materials is the practical engineering design process in which high performance shield materials are incorporated into cost effective designs. Many of these engineering issues for use of polymeric composites are already being addressed in the development of light weight aircraft designs. Practical engineering experience gained in the use of polymeric composites in aircraft design is expected to have an important impact on the future of spacecraft design. 


\section{REFERENCES}

1. G. D. Badhwar, F. A. Cucinotta, P. M. O'Neill, Radiat. Res. 134, P. 9 (1993).

2. D. F. Smart, M. A. Shea, J. Spacecraft and Rockets 26, p. 403 (1989).

3. J. W. Wilson, F. M. Denn, Preliminary Analysts of the Implications of Natural Radiations on Geostationary Operations. NASA TN D-8290, 1976.

4. E. G. Stassenopolous in High-energy Radiation Background in Space, edited by A. C. Rester, J. I. Trombka, AIP Conference Proceedings 186, New York, p. 3, 1986.

5. H. Wu, W. Atwell, F. A. Cucinotta, C. Yang, Estimate of Space Radiation-Induced Cancer Risks for International Space Station. NASA TM-104818, 1996.

6. National Council on Radiation Protection, Guidance on Radiation Received in Space Activities. NCRP Report No. 98, 1989.

7. National Academy of Science, Radiation Hazards to Crews of Interplanetary Missions: Biological Issues and Research Strategies. NAS Press, Washington DC, 1996.

8. J. W. Wilson, J. Miller, A. Konradi, F. A. Cucinotta, eds., Shielding Strategies for Human Space Exploration, NASA CP-3360, 1997.

9. R. A. Mewalt, Interplanetary Particle Environment, Publ. 88-28, Jet Propulsion Laboratory, Pasadena, p. 112, 1988.

10. J. A. Simpson, Ann. Rev. Nucl. Part. Sci. 33 p. 323 (1983).

11. International Commission on Radiological Protection, 1990 Recommendations of the ICRP, ICRP Publication No. 60, Pergamon Press, 1991.

12. J. W. Wilson, F. A. Cucinotta, J. L. Shinn, Cell Kinetics and Track Structure, Biological Effects and Physics of Solar and Galactic Cosmic Radiation, Part A, eds. C. E. Swenberg et al., Press, pp. 295-338, 1993.

13. T. C. Yang, L. M. Craise, Biological Response to Heavy Ion Exposures. In Shielding Strategies for Human Space Exploration. Eds. J. W. Wilson et al. Chpt. 6, pp. 91-109, NASA CP 3360, 1997.

14. F. A. Cucinotta, J. W. Wilson, R. Katz, Int. J. Radiat. Biol. 69, p. 593 (1995).

15. F. A. Cucinotta, J. W. Wilson, Phys. Med. Biol. 39, p. 1811 (1994).

16. E. L. Alpen et al. Radiat. Res. 136, p. 382 (1993), Adv. Sp. Res. 14, p. 573 (1994).

17. F. A. Cucinotta, et al. Computational Procedures and Database Development. In Shielding Strategies for Human Space Exploration. Eds. J. W. Wilson et al. Chpt. 8, pp. 91-109, NASA CP 3360, 1997.

18. H. Tai et al. Comparison of stopping power and range databases for radiation transport study. NASA TP 3644, 1997.

19. J. Miller et al. Acta Astronautica 42, p. 389 (1998).

20. J. W. Wilson et al. Adv. Space Res. 14(10), p. 841 (1994). 
21. R. G. Alsmiller, D. C. Irving, W. E. Kinney, and H. S. Moran, "The validity of the straightahead approximation in space vehicle shielding studies," In Second Symposium on Protection Against Radiations in Space. A. Reetz, ed. NASA SP-71, pp. 177-181; 1965.

22. J. W. Wilson et al., HZETRN: Description of a free-space ion and nucleon transport and shielding computer program. NASA TP 3495, 1995.

23. J. W. Wilson et al. Health Phys. 68, p. 50 (1995).

24. J. R. Letaw, R. Silberberg, and C. H. Tsao, "Radiation haxards on space missions outside the magnetosphere." Adv. Space Res. 9(10), p. 285 (1989).

25. J. W. Wilson, L. W. Townsend, and F. F. Badavi, "A semiempirical nuclear fragmentation model." Nucl. Inst. Methods Phys Res. B18, p. 225 (1987).

26. J. W. Wilson, J. L. Shinn, L. W. Townsend, R. K. Tripathi, F. F. Badavi, and S. Y. Chun, "NUCFRG2: A semiempirical nuclear fragmentation model." Nucl. Inst. Methods Phys Res. B94, p. 95 (1995).

27. F. A. Cucinotta, L. W. Townsend, J. W. Wilson, J. L. Shinn, G. D. Badhwar, and R. R. Dubey, "Light ion components of the galactic cosmic rays: Nuclear interactions and transport theory." Adv. Space Res. 17(2), p. 77 (1996). 